\title{
Prevention by the pound
}

Previously published at www.cmaj.ca

${ }^{6} \mathrm{~T}$ his is new to me, I was a really healthy person," she said, sitting in the tiny exam room. Her comment stopped me, mid busy day. She was an older woman, in her 80s, but seemed fit and healthy. A year ago, physicians had "picked up on all these things" — her hypertension, her cholesterol, her atrial fibrillation - and started treating them all. The thing was, nothing had actually happened to her over the past year, nothing had changed. She hadn't had a heart attack or a stroke. Her exercise capacity was about the same. But now she had to take a handful of pills each day; she had to go to the lab once every few weeks to monitor her anticoagulation. She'd joined the ranks of the chronically ill, even though she wasn't actually sick. Or maybe she was. Or maybe she would have been.

This is exactly as it should be in the age of preventive medicine. But patients like her make me wonder if in trying to keep people well, we've made them feel unwell. For the most part these risk factors are asymptomatic aspects of physiology, but they've come to be understood as disease states in their own right - and patients thus treated have come to feel as though they are diseased.

"First, do no harm." It's one of our most basic obligations as physicians. How do we rationalize this tenet in the context of preventive medications? Notwithstanding its many flaws, the biomedical disease/treatment model at least implies addressing conditions that are actually causing or will cause suffering for an individual. In that scenario, the physician can say to the patient "Yes, I believe this intervention will help you." But preventive medicine treats individuals based on risks being managed at the population level. There's no way to know if the individual patient will benefit.

And yet it seems that the average patient on preventive medications assumes that the pills they take will help

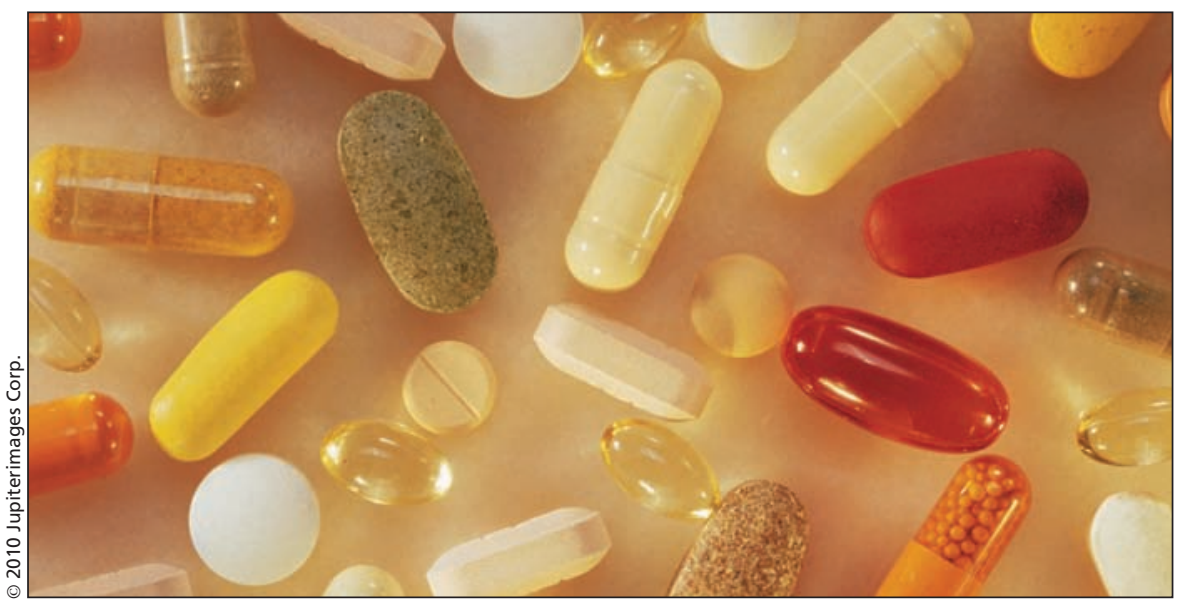

them. Certainly, people don't take their antihypertensive and lipid lowerer and then say "I hope I am the one out of 20 to 100 taking this medication for $\mathrm{x}$ years that will have a catastrophic event prevented ..." How information is presented goes a long way in shaping a patient's understanding. For example, if the doctor says to the patient, "Based on your blood pressure and cholesterol readings and according to these guidelines, you should take one of these medications," it appears to be a fairly well-defined matter. But if I extend this discussion, I could talk about the probability of concrete benefit for an individual. "If there were 20 or 30 or more of you, the chances would be that one of you would benefit. But all would be subject to the medication's potential side effects. All would take a new medication every day, probably for the rest of their lives." Suddenly, whether or not to take the medication is markedly less clear.

"Compliance is always the big problem," said the drug representative who stopped by the office to show us the new lipid guidelines. Even though we're trying to move beyond it, that kind of sentiment still exists within the medical culture. We know what's right for people because we have evidence for it, and if people don't want to cooperate, it's because they're irrational. Leaving aside that many of the decisions we make are based on how we feel, and there's not necessarily anything wrong with that, there seems to be a certain rationality in reluctance to take preventative medications. Ultimately we don't know what's going to happen to us. We don't know how long we've got. To want to simply feel well when we feel well is a natural tendency, and taking pills each day detracts from this.

Perhaps the patient who said to me "I used to be really healthy" had been prevented from experiencing a crippling stroke over the past year, there's about a $5 \%$ chance that she would have. Do the unknown successes justify the known sequellae, biological, psychological, and cultural? In the end, I asked this patient "Would you just rather not take all these pills?" She said "Oh no, I'll take them, I have to take them." It's the right answer from the public health point of view and part of me agrees with it. But part of me wonders if I'll take them myself when the time comes - or if I'll just decline the tests, and live not knowing, and see what happens.

\section{Patrick Sullivan MD}

General practitioner

Halifax, NS

Do you have an opinion about this article? Post your views at www.cmaj.ca. Potential Salon contributors are welcome to send a query to salon@cmaj.ca. 